TITLE:

\title{
Alpha-band desynchronization in human parietal area during reach planning.
}

\section{$\operatorname{AUTHOR}(\mathrm{S}):$}

Fumuro, Tomoyuki; Matsuhashi, Masao; Miyazaki, Tomoko; Inouchi, Morito; Hitomi, Takefumi; Matsumoto, Riki; Takahashi, Ryosuke; Fukuyama, Hidenao; Ikeda, Akio

\section{CITATION:}

Fumuro, Tomoyuki ... [et al]. Alpha-band desynchronization in human parietal area during reach planning.. Clinical neurophysiology 2015, 126(4): 756-762

\section{ISSUE DATE:}

2015-04

URL:

http://hdl.handle.net/2433/200243

\section{RIGHT:}

(c) 2014. This manuscript version is made available under the CC-BY-NC-ND 4.0 license http://creativecommons.org/licenses/by-nc-nd/4.0/.; この論文は出版社版でありません 。引用の際には出版社版をご確認ご利用ください。; This is not the published version. Please cite only the published version. 


\section{Title}

Alpha-band desynchronization in human parietal area during reach planning

\section{Authors}

Tomoyuki Fumuro ${ }^{1,2}$, Masao Matsuhashi ${ }^{2,3}$, Tomoko Miyazaki ${ }^{4}$, Morito Inouchi ${ }^{4,5}$, Takefumi Hitomi ${ }^{4,5,6}$, Riki Matsumoto ${ }^{1}$, Ryosuke Takahashi ${ }^{4}$, Hidenao Fukuyama ${ }^{3}$, Akio Ikeda ${ }^{1, *}$

\section{Affiliations}

${ }^{1}$ Department of Epilepsy, Movement Disorders and Physiology, Kyoto University Graduate School of Medicine

54 Shogoin-kawaracho, Sakyo-ku, Kyoto, 606-8507, JAPAN

${ }^{2}$ Research and Educational Unit of Leaders for Integrated Medical System

54 Shogoin-kawaracho, Sakyo-ku, Kyoto, 606-8507, JAPAN

${ }^{3}$ Human Brain Research Center, Kyoto University Graduate School of Medicine 54 Shogoin-kawaracho, Sakyo-ku, Kyoto, 606-8507, JAPAN

${ }^{4}$ Department of Neurology, Kyoto University Graduate School of Medicine 54 Shogoin-kawaracho, Sakyo-ku, Kyoto, 606-8507, JAPAN

${ }^{5}$ Department of Respiratory Care and Sleep Control Medicine, Kyoto University Graduate School of Medicine

54 Shogoin-kawaracho, Sakyo-ku, Kyoto, 606-8507, JAPAN

${ }^{6}$ Department of Clinical Laboratory Medicine, Kyoto University Graduate School of Medicine

54 Shogoin-kawaracho, Sakyo-ku, Kyoto, 606-8507, JAPAN

\section{*Corresponding author}

Akio Ikeda, M.D., Ph.D.

Department of Epilepsy, Movement Disorders and Physiology, Kyoto University Graduate School of Medicine

54 Shogoin-kawaracho, Sakyo-ku, Kyoto, 606-8507, JAPAN

E-mail: akio@kuhp.kyoto-u.ac.jp

Tel: (+81)-75-751-3662, Fax: (+81)-75-751-3663 


\section{Keywords}

Alpha-band desynchronization; Reaching; Parietal area; Motor planning;

Number of pages: 39 (include figures); Number of reference: 32; Title characters: 73;

Number of figures: 3; Number of tables: 0; Abstract words: 167; Text words: 4647

(include references); Key words: 4 


\section{Acknowledgements}

The authors declare no competing financial interests.

This work was supported by Grants-in-Aid for Scientific Research (B) 26282218, 26293209, (C) 26330175 from the Ministry of Education, Culture, Sports, Science and Technology of Japan (MEXT). 


\section{Highlight}

- Posterior parietal cortex (PPC) uses frequency-specific dynamics in planning visuo-motor goal-directed tasks.

- Upper alpha band PPC activity codes for preparing goal-directed actions, whereas lower alpha band PPC activity reflects general task demands and attentional processes that are not task-specific.

- PPC activates earlier in a goal-directed action. 


\section{Abstract}

Objective: The symptoms with optic ataxia suggest that simple and visually guided hand movements are controlled by 2 different neural substrates. To assess the differential frequency-coded posterior parietal cortex (PPC) role in planning visuo-motor goal-directed tasks, we studied the action specificity of event-related desynchronization (ERD) in this area.

Methods: We investigated cortical activity by electroencephalography, while 16 healthy subjects performed self-paced reaching or wrist extension (control) movements. Time-frequency representations were calculated for each movement during the preparatory period.

Results: ERD dynamics in upper alpha-band indicated that preparing a goal-directed action activates contralateral PPC to the moving hand around $1.2 \mathrm{~s}$ before starting the movement, while this activation is later (around $0.7 \mathrm{~s}$ ) in preparing a not-goal-directed action. The posterior dominant rhythm had peak frequency of lower alpha-band at bilateral parietal.

Conclusions: Posterior parietal cortex encodes goal-directed movement preparation through upper alpha-band activity, whereas general attention is processed via lower alpha-band oscillations.

Significance: Preparing to reach an object engages posterior parietal cortex earlier than a not-goal directed movement. 


\section{Introduction}

Reaching for an object is a type of complex hand movement performed by humans and primates, and is often called visually guided reaching or praxis. We reach for objects with an incredibly high degree of precision in daily life. Such behavior appears to be effortless, even with unexpected perturbations such as an object relocation (Prablanc and Martin, 1992; Pisella et al., 2000). However, reaching movements require integrated information regarding the object's position and orientation to guide the hand to the object with accuracy, whereas information regarding the object's shape and size determines how the fingers move opposite to the thumb to grasp the object. Recent functional imaging studies that used functional MRI or PET in humans have revealed that the posterior parietal cortex (PPC) plays an important role in controlling praxis movements by continuously integrating sensory information regarding the body state and environment (Culham et al., 2006). However, these modern techniques provide temporal resolution that is insufficient for reliable quantitative analysis of activation times.

In humans and primates, motor-related activity has been successfully investigated through electroencephalographic (EEG) oscillatory activity analysis. It is well known that event-related desynchronization (ERD) in the alpha-band $(8-13 \mathrm{~Hz})$ starts about $1.5 \mathrm{~s}$ before the onset of movement (Pfurtscheller and Berghold, 1989). This activity is presumed to reflect cortical activity related to movement planning (Pfurtscheller and Lopes da Silva, 1999). Experimental data suggest that alpha ERD represents an electrophysiological correlate of activated cortical areas that is related to information processing, selective attention, and motor preparation (Van Winsum et al., 1984; Pfurtscheller, 1992; Dujardin et al., 1993, 1995). Furthermore, Pfurtscheller et al. (2000) reported that in a motor task, the upper frequency mu rhythms $(10-12 \mathrm{~Hz})$ reflects a more somatotopic spatial ERD pattern than the lower frequency mu rhythms$(8-10 \mathrm{~Hz})$. This different behavior between the lower and upper alpha-band components indicates that the lower alpha ERD reflects general task demands and attentional processes that are not task-specific, whereas the upper alpha ERD develops when movement-related information is processed; therefore, it is task-specific (Pfurtscheller et al., 2000). In addition, the posterior dominant rhythm (PDR) is an idling rhythm, indicative of a relative decrease in conscious attention or visual processing 
(Pfurtscheller and Aranibar, 1977). Approximately $80 \%$ of healthy adults had a PDR between 9 and $11 \mathrm{~Hz}$ (Kellaway, 1990).

Movement-specific ERD has been recorded not only from scalp electrodes but also subdural electrodes (Toro et al., 1994). However, only a few investigations have used this approach to study more practical and coordinated movements such as reaching, catching, or grasping (Tombini et al., 2009; Van Der Werf et al., 2010; Virji-Babul et al., 2010).

The present study aimed to clarify the involvement of PPC in movement planning and execution by revealing upper alpha-band ERD in parietal area, when reaching for an object (i.e., target- and body-related movements). Therefore, we compared reaching and simple movements to determine whether the underlying neural sources of EEG activity for these movements can be distinguished as independent. 


\section{Materials and Methods}

\subsection{Participants}

Subjects were 16 healthy right-handed university students (6 men, 10 women; age, 22-25 years) with normal or corrected-to-normal vision and with no reported history of neurological or psychiatric illnesses. All subjects provided an informed consent for participating in the study. The Ethical Committee of Kyoto University approved the experimental protocol (No. E-929).

\subsection{Recording conditions}

EEG signals from $23 \mathrm{Ag} / \mathrm{AgCl}$ surface electrodes placed on the scalp were recorded according to the 10-10 International System. For the quantitative analysis, 5 regions of interest were defined (Figure 1), which included the left fronto-central (F3, FC1, FC3, C1, C3); right fronto-central (F4, FC2, FC4, C2, C4); midline (Fz, FCz, Cz); left parietal area (CP3, P3, P7, PO3, PO7); and right parietal area (CP4, P4, P8, PO4, PO8). Electromyograph (EMG) activity was recorded simultaneously with EEG activity from a pair of $\mathrm{Ag} / \mathrm{AgCl}$ surface electrodes placed $3 \mathrm{~cm}$ apart over the left and right deltoid (DEL) and extensor carpi radialis muscles (ECR). Two channels of electrooculograms (EOG) were recorded horizontally (HEOG) at the external canthi of both eyes and vertical EOG (VEOG) at the upper and lower edges of the left eye. EEG, EOG, and EMG findings were recorded using a DC-EEG system (NEURO PRAX, neuroconn GmbH, Ilmenau, Germany). This DC-EEG system enables us to record without a low cut filter as done previously (Fumuro et al., 2013). EEG electrodes were referred for average signal recorded between the left and right mastoids leads. The EEG, EOG, and EMG signals were acquired at a sampling frequency of $4.096 \mathrm{KHz}$ with a 1.2 $\mathrm{KHz}$ high-cut filter. The impedance of all electrodes was kept less than $5 \mathrm{k} \Omega$ as also done previously in this system (Fumuro et al., 2013).

\subsection{Experimental procedures}

During the EEG recording, subjects were seated comfortably in an armchair with their hands resting on a pillow. They were instructed to observe a cup (diameter 9 $\mathrm{cm}$, height $7 \mathrm{~cm}$ ) located approximately $50 \mathrm{~cm}$ in front of them. They were asked to reach and hold the handle of the cup with one hand in a brisk (less than $1 \mathrm{~s}$ ), self-paced movement, and to perform approximately one reach every $10 \mathrm{~s}$. The movement interval 
varied, and it never fixed as $10 \mathrm{sec}$ of interval. Each subject decided the movement interval freely without any external trigger. As a control, subjects performed a simple wrist extension with the same timing and frequency as the reaching movement. Subjects practiced the task before the recording until their performance was satisfactory.

Subjects conducted the reaching and wrist extension tasks in a set of 20 trials, and performed approximately 6 sets of each task with each hand. In reaching and control tasks, the same side of hand was employed to perform a set of each task and the other side of hand was alternatively used for each set, with a short interval to rest between the sets. Overall, subjects performed 4 different tasks within a single set: reaching with the right $(\mathrm{Rr})$ or left hand $(\mathrm{Rl})$ and the control with the right $(\mathrm{Cr})$ or left hand $(\mathrm{Cl})$. These tasks were performed in one of the 4 following sequences: 1$) \mathrm{Rr} \rightarrow \mathrm{Rl}$ $\rightarrow \mathrm{Cr} \rightarrow \mathrm{Cl}$, 2) $\mathrm{Rl} \rightarrow \mathrm{Rr} \rightarrow \mathrm{Cl} \rightarrow \mathrm{Cr}, 3) \mathrm{Rr} \rightarrow \mathrm{Cr} \rightarrow \mathrm{Rl} \rightarrow \mathrm{Cl}$, or 4) $\mathrm{Rl} \rightarrow \mathrm{Cl} \rightarrow \mathrm{Rr} \rightarrow$ Cr. The 16 patients were randomly assigned into 4 groups $(n=4)$, with each group performing one of the above 4 task patterns. Subjects were instructed to minimize their shoulder, trunk, and head movements. The examiner observed the subject throughout the procedure to confirm a proper execution of motor task periods. During the examination, EOG and EMG were monitored closely to ensure the degree of subject's vigilance and performance.

After performing the movement tasks, subjects underwent additionally EEG recording for approximately $1-3$ min while resting with their eyes-closed. The EEG recordings were used to measure the PDR.

\subsection{Analysis}

Data were processed offline using custom-written MATLAB (MathWorks, Natick, MA, USA) scripts (by MM, one of the authors). The data were filtered with a $60-\mathrm{Hz}$ notch filter. A time-frequency analysis was performed to identify the time course of oscillatory changes before voluntary movements. The movement onset was determined by the initiation of the first EMG burst recorded from either DEL or ECR. We analyzed the EEG data recorded before the movement onset in 3-s epochs. Epochs containing artifacts were excluded from the analysis. Artifacts included muscle activity between movements (artifacts occurring in the $5 \mathrm{~s}$ preceding movement onset that exceeded a peak-to-peak amplitude of $50 \mu \mathrm{V}$ in the EMG, or $\pm 50 \mu \mathrm{V}$ in the EEG channel with a $30-\mathrm{Hz}$ low-frequency filter), blink contamination in the analysis window 
(occurring in the $3.5 \mathrm{~s}$ preceding movement onset, EOG peak-to-peak amplitude exceeding $200 \mu \mathrm{V}$ ), and inter-trial intervals shorter than $6 \mathrm{~s}$. If the interval between the initial EMG bursts in DEL and ECR exceeded $200 \mathrm{~ms}$, the trial in which the EMG data were recorded was considered to include incorrect task performance and was rejected from further analysis. Event-related potentials (ERPs) were calculated by averaging the remaining trials. Data recorded from 3000 to $2000 \mathrm{~ms}$ before the movement onset were used as the baseline. An averaged data of less than 50 trials for a movement task pattern was excluded from further analysis.

The time-frequency representation of EEG power was calculated using a short-time Fourier transformation (STFT) with a Hanning window of $500 \mathrm{~ms}$ (frequency resolution of $2 \mathrm{~Hz}$ ) incremented in $100 \mathrm{~ms}$ steps. The data were averaged across trials and transformed by the $\log$ of 10 ; the baseline power was subtracted frequency-wise from the log-transformed data to obtain the power change relative to the baseline in log scale.

The onset of ERD was defined as the first point before the movement onset at which the log of power was at least 3 standard deviations (SD) less than the baseline period for at least $300 \mathrm{~ms}$. In the frequency of beta band, there were not a sufficient number of subjects who provided the qualified ERD that met the above criteria for the ERD onset. Thus, we focused on the alpha band ERD in this study.

The relative power in $12 \mathrm{~Hz}$ at $800 \mathrm{~ms}$, which represents $\pm 250 \mathrm{~ms}$ (from 1050 to $550 \mathrm{~ms}$ before movement onset) in the STFT analysis, was used in the statistical analysis for the following reasons: 1) The ERD of the upper (10-12 Hz) alpha rhythm could be more localized, and was considered to exhibit greater specificity for the type of movement pattern than the lower (8-10 Hz) alpha rhythm (Pfurtscheller et al., 2000). 2) Alpha ERD becomes more significant closer to the time of movement onset (Pfurtscheller and Berghold, 1989). Therefore, the time window from 1050 to $550 \mathrm{~ms}$ pre-movement onset would detect a greater contralateral preponderance of alpha ERD.

In order to increase the signal to noise ratio, we grouped the EEG channels into 5 groups (Figure 1). To compare the data recorded during reaching and wrist extensions, we calculated a representative grand-average ERD power based on each of electrode-oriented 5 groups, and labeled their anatomical position as either contralateral or ipsilateral to the moving hand. Thus, trials were analyzed according to their contralateral or ipsilateral designation, rather than the left and right hand. The values for 
5 locations (contralateral fronto-central, midline, ipsilateral fronto-central, contralateral parietal area, and ipsilateral parietal area) were statistically analyzed.

To identify the peak frequency and its distribution of PDR, segments of EEG recorded during complete conscious, without substantial artifact, were subjected to a quantitative analysis, including a fast Fourier transform (FFT) power spectral analysis (Hanning window of $1024 \mathrm{~ms}$ at frequency resolution of $1 \mathrm{~Hz}$ ). The mean PDR frequency was measured from the averaged power spectral density estimate across all subjects.

\subsection{Statistics}

Statistical analyses were performed using commercially available statistical software (SPSS 15.0 J for Windows; SPSS, Chicago, IL, USA). To determine whether ERD patterns differed between reaching and control movements, we performed a three-way repeated-measures ANOVA. The within-subject factors were Hand (left and right); Region (contralateral fronto-central, midline, ipsilateral fronto-central, contralateral parietal area, and ipsilateral parietal area); and Movement (reaching and wrist extension). A paired $t$-test was used to examine the ERD onset in the contralateral parietal area. One-way ANOVA with repeated measures was used to determine the laterality of ERD for the parietal areas in each movement. To clarify the laterality of ERD in each movement type regardless of the moving hand, the lateralized ERD index (LERDI) was computed from the difference in ERD power between the contralateral and ipsilateral parietal areas by the double subtraction method (Smid et al., 1987; de Jong et al., 1988): LERDI = [(left parietal - right parietal $)$ right hand $+($ right parietal left parietal) left hand]/2. The significance level was set at $\mathrm{P}<0.05$. 


\section{Results}

Data from a subject was excluded from the statistical analysis because an insufficient number of trials were recorded. In addition, data from another subject including significant artifacts during left wrist extensions was excluded.

Figure $2 A$ presents grand-average mapped spatially enhanced alpha ERD for reaching and wrist extension. In the grand-averaged ERD, the $12-\mathrm{Hz}$ frequency band revealed the highest power decreases relative to baseline (Figure $2 A$ ). A three-way repeated-measures ANOVA revealed a significant effect of Region $(\mathrm{F}(2.116,27.509)=$ $17.852, \mathrm{P}<0.001)$ for the 12-Hz ERD. Significant interactions were observed between two factors: Hand $\times$ Region $(F(4,52)=5.210, \mathrm{P}=0.001)$ and Region $\times$ Movement $(\mathrm{F}(1.648,21.429)=7.894, \mathrm{P}=0.004)$. The former finding indicated that the ERD lateralization toward the contralateral hemisphere to the moving hand was significantly dominant before the right hand movement as compared with the left hand movement (Figure $2 B$ ). The latter finding indicated the maximum of 12-Hz ERD was observed in the more posterior parietal areas during reaching preparation, and was more widely distributed contralaterally than ipsilaterally.

In reaching task, the grand-average ERD value indicated the upper alpha (maximum at $12 \mathrm{~Hz}$ ); ERD initiated at least $1 \mathrm{~s}$ before reaching and revealed the maximum value in the contralateral parietal area, and to a lesser degree in the contralateral fronto-central and ipsilateral parietal areas (Figure $2 A$ Upper).

In wrist extension task, the grand-average ERD value indicated the upper alpha (maximum at $12 \mathrm{~Hz}$ ) ERD started between 0.5 and $1 \mathrm{~s}$ before wrist extension, and revealed the maximum value localized in the anterior contralateral parietal area. A clear ERD was not identified in the ipsilateral hemisphere (Figure $2 A$ Lower).

Figure $2 C$ presents the grand-average amplitude of LERDI, which indicated the $12-\mathrm{Hz}$ ERD occurring in both parietal areas, from 1050 to $550 \mathrm{~ms}$ before the movement onset, were mostly biased to the contralateral hemisphere. This finding is consistent with movement planning for the contralateral hand. A strong lateralization was suggested by the LERDI values for reaching $(-1.1 \pm 1.7)$ and wrist extension $(-1.2$ $\pm 1.4)$. One-way ANOVA with repeated-measures revealed a significantly higher LERDI over the contralateral parietal area prior to both reaching and wrist extension $(\mathrm{F}(1,13)=16.300, \mathrm{P}=0.001)$, whereas it failed to reveal a significant difference in the mean LERDI between reaching and wrist extension. 
In the contralateral parietal area, data for 10 of 15 subjects met the criteria for ERD onset with right hand reaching and wrist extension, whereas data for only 4 of 14 subjects met the criteria for left hand movements. Therefore, a total of 14 subject's data (10 from right hand task and 4 from left hand task) were combined and statistically analyzed. The mean onset of the ERD in the contralateral parietal area was $-1.2 \pm 0.5 \mathrm{~s}$ (mean $\pm \mathrm{SD}$ ) for reaching and $-0.7 \pm 0.4 \mathrm{~s}$ for wrist extension. A paired $t$-test indicated a significant and early ERD onset over the contralateral parietal area prior to reaching, when compared with wrist extension $(\mathrm{t}=2.424, \mathrm{df}=13, \mathrm{P}=0.031$; Figure $2 D)$.

Unlike the contralaterally biased $12-\mathrm{Hz}$ ERD, the PDR was distributed bilaterally and more posteriorly, but was slightly biased for the right side (Figure 3). These findings indicate the ERD and PDR induced by the task movements differed in their peak frequency and distribution. 


\section{Discussions}

The aim of this study was to determine the temporal characteristics and spatial distribution of brain regions activated during the planning of reaching and wrist extension movements. We identified 3 main differences between these 2 movement conditions in the ERD analysis. The first difference was in the distribution of $12-\mathrm{Hz}$ ERD that revealed a task-specific pattern (Figures $2 A$ and 3 ). We estimated the upper alpha-band activity involved in reach planning was located in the parietal area contralateral to the moving hand. In contrast, ERD was localized primarily to the anterior region of the contralateral parietal area for wrist extension. These findings agree with the findings reported in the monkey literature that describe task-specific movement-goal representations in the PPC; neural spiking activity and local field potential recordings in the monkey PPC have revealed separate subregions for reaching and simple hand movements: the parietal reach region for reach planning (Snyder et al., 1997; Galletti et al., 2003; Scherberger et al., 2005; Scherberger and Andersen, 2007) and the primary sensorimotor area for simple hand movements (Murthy and Fetz, 1996; Baker et al., 1997; Courtemanche and Lamarre, 2005). The second difference was the alpha ERD appeared earlier in the contralateral parietal area for reaching (1.2 s before movement onset) than for wrist extension ( $0.7 \mathrm{~s}$ before movement onset; Figure $2 D)$. The difference of $0.5 \mathrm{~s}$ may be interpreted as the time required to perform the required visuomotor transformation ensuring an accurate performance. The third difference was the enlarged distribution and locations within the ipsilateral and contralateral parietal areas during the reach preparation that was consistent with previous findings (Figure $2 \mathrm{~A}$ Upper) (Calton et al., 2002; Prado et al., 2005). This supports the idea that the ipsilateral parietal area could be involved in the executive control and planning of reaching movements (Naranjo et al., 2007). Considering together, these results support our hypothesis that the PPC is engaged in the preparation of praxis movements. An earlier onset and enlarged distribution of 12-Hz ERD could be essential for complex tasks in which the hand is moved during reaching, because the purpose of the movement is processed to shape the hand in the appropriate manner in the premotor and motor cortices.

While the anatomical divisions and functions of these cortical areas have been evaluated in primates, it is difficult to equate their anatomical locations to those for the human subjects in the present study because of the lack of spatial precision offered by 
scalp-recorded EEG. This method is particularly useful to assess temporal changes in cortical activity; however, it lacks the spatial accuracy of neuroimaging methods. In particular, scalp-recorded EEG does not provide a direct recording from the cortical surface, but is recorded over the scalp and is separated from the brain by the meninges, skull, and skin. Therefore, we attempted to clearly delineate distinguishable distribution patterns of ERD between reaching and wrist extension movements. Nevertheless, localization of the reaching-induced alpha ERD would be consistent with a subdural EEG recording of a movement-related cortical potential (MRCP) in the superior parietal lobule (SPL), inferior parietal lobule (IPL), and peri-intra parietal sulcus during reaching movements (Inouchi et al., 2013). These areas previously have been implicated in the planning of reaching movements (Calton et al., 2002; Prado et al., 2005; Naranjo et al., 2007). This is also supported by the other subdural EEG recordings from primary sensorimotor area that have revealed the alpha ERD and MRCP share features in time and source space (Toro et al., 1994). However, alpha ERD was not always detected at cortical sites generating MRCP (Arroyo et al., 1993). Therefore, the correlation of ERD and MRCP are still controversial.

Figures $2 B$ and 3 suggested that contralateral dominance of ERD was clearly observed before right hand movements, whereas the ERD before left hand movements site bilateral hemispheres. This finding is consistent with the previous study (Bai et al., 2005).

Herein, we compared the temporal evolution of the alpha ERD using a modern high-resolution EEG technique. Other frequency bands were not considered because simple motor tasks do not evoke a gamma band response (i.e., $40 \mathrm{~Hz}$ ), and post-movement beta band rebound is not associated with a stable hand positioning, particularly in reaching sets. The distribution of alpha ERD in the parietal area is an important finding that should be carefully discussed. A strong suppression of alpha rhythms in the parietal area could result from attentional requirements of visuomotor tasks (Dujardin et al., 1993). However, clearly different patterns of alpha modulation between ERD and PDR explain the distinct neural systems underlying the motor preparation and general attention. Namely, the goal directed and not-goal directed actions are crucially different.

Figure 3 shows the grand-average PDR comprises from the peak frequency of $10 \mathrm{~Hz}$ at posterior parietal site bilaterally. In contrast, reaching representations were 
indicated by power decreases in a $12-\mathrm{Hz}$ alpha frequency band (Figure $2 \mathrm{~A} U p p e r$ ), and this representation was further emphasized with significant lateralization to the contralateral hemisphere for reaching hand movement (Figures $2 C$ and 3). Thus, we found a clear difference in the distribution of the peak frequency between reaching and PDR. Furthermore, upper alpha ERD shows a movement-type specific pattern, clearly different between visuo-motor goal directed task and simple hand movement. These findings strengthen the distinction of neuronal activity between the lower alpha-band reflecting attentional processes that are not task-specific and the upper alpha-band develops when specific movement-related information is processed. 


\section{Conclusions}

In conclusion, the present EEG investigation supports the working hypothesis that alpha ERD in the parietal area provides complementary information on brain activity in humans during the preparation and execution of volitional reaching movements. Our main finding was that alpha ERD originating from the parietal area encodes the reaching movement, whereas alpha ERD originating from fronto-central (e.g, suppression of the mu rhythm) and the anterior region of the parietal area encodes wrist extension. These results, suggest that distinct modules in the parietal cortex encode different movements through selective alpha-band desynchronization, which are compatible with the functional organization of the PPC. In future studies, these findings are useful in testing other hypotheses, such as the relationships between movement complexity, and specific symptoms due to abnormality of dorsal pathway and temporal neurological change in PPC. A further refinement using a high spatial resolution analysis, such as magnetoencephalography and high density EEG, will provide more insights to understand the function of local and global alpha-band oscillation. 


\section{References}

Arroyo S, Lesser RP, Gordon B, Uematsu S, Jackson D, Webber R. Functional significance of the mu rhythm of human cortex: an electrophysiologic study with subdural electrodes. Electroencephalogr Clin Neurophysiol 1993;87:76-87.

Bai O, Mari Z, Vorbach S, Hallett M. Asymmetric spatiotemporal patterns of event-related desynchronization preceding voluntary sequential finger movements: a high-resolution EEG study. Clin Neurophysiol 2005;116:1213-21.

Baker SN, Olivier E, Lemon RN. Coherent oscillations in monkey motor cortex and hand muscle EMG show task-dependent modulation. J Physiol 1997;501:225-41.

Calton JL, Dickinson AR, Snyder LH. Non-spatial, motor-specific activation in posterior parietal cortex. Nat Neurosci 2002;5:580-8.

Courtemanche R, Lamarre Y. Local field potential oscillations in primate cerebellar cortex: synchronization with cerebral cortex during active and passive expectancy. J Neurophysiol 2005;93:2039-52.

Culham JC, Cavina-Pratesi C, Singhal A. The role of parietal cortex in visuomotor control: what have we learned from neuroimaging? Neuropsychologia 2006;44:2668-84.

de Jong R, Wierda M, Mulder G, Mulder LJ. Use of partial stimulus information in response processing. J Exp Psychol Hum Percept Perform 1988;14:682-92.

Dujardin K, Bourriez JL, Guieu JD. Event-related desynchronization (ERD) patterns during memory processes: effects of aging and task difficulty. Electroencephalogr Clin Neurophysiol 1995;96:169-82.

Dujardin K, Derambure P, Defebvre L, Bourriez JL, Jacquesson JM, Guieu JD. Evaluation of event-related desynchronization (ERD) during a recognition task: effect of attention. Electroencephalogr Clin Neurophysiol 1993;86:353-6.

Fumuro T, Matsuhashi M, Mitsueda T, Inouchi M, Hitomi T, Nakagawa T, et al. Bereitschaftspotential augmentation by neuro-feedback training in Parkinson's disease. Clin Neurophysiol 2013;124:1398-405.

Galletti C, Kutz DF, Gamberini M, Breveglieri R, Fattori P. Role of the medial parieto-occipital cortex in the control of reaching and grasping movements. Exp Brain Res 2003;153:158-70. 
Inouchi M, Matsumoto R, Taki J, Kikuchi T, Mitsueda-Ono T, Mikuni N, et al. Role of posterior parietal cortex in reaching movements in humans: Clinical implication for 'optic ataxia'. Clin Neurophysiol 2013;124:2230-41.

Kellaway P. An orderly approach to visual analysis: characteristics of the normal EEG of adults and children. In: Daly DD, Pedley TA, Editors. Current Practice of Clinical Electroencephalography, 2nd Edition: Raven Press, New York, 1990: 139-99.

Murthy VN, Fetz EE. Oscillatory activity in sensorimotor cortex of awake monkeys: synchronization of local field potentials and relation to behavior. J Neurophysiol 1996;76:3949-67.

Naranjo JR, Brovelli A, Longo R, Budai R, Kristeva R, Battaglini PP. EEG dynamics of the frontoparietal network during reaching preparation in humans. Neuroimage 2007;34:1673-82.

Pfurtscheller G. Event-related synchronization (ERS): an electrophysiological correlate of cortical areas at rest. Electroencephalogr Clin Neurophysiol 1992;83:62-9.

Pfurtscheller G, Aranibar A. Event-related cortical desynchronization detected by power measurements of scalp EEG. Electroencephalogr Clin Neurophysiol 1977;42:817-26.

Pfurtscheller G, Berghold A. Patterns of cortical activation during planning of voluntary movement. Electroencephalogr Clin Neurophysiol 1989;72:250-8.

Pfurtscheller G, Lopes da Silva FHL. Event-related EEG/MEG synchronization and desynchronization: basic principles. Clinical Neurophysiology 1999;110:1842-57.

Pfurtscheller G, Neuper C, Krausz G. Functional dissociation of lower and upper frequency mu rhythms in relation to voluntary limb move ment. Clin Neurophysiol 2000;111:1873-9.

Pisella L, Grea H, Tilikete C, Vighetto A, Desmurget M, Rode G, et al. An 'automatic pilot' for the hand in human posterior parietal cortex: toward reinterpreting optic ataxia. Nat Neurosci 2000;3:729-36.

Prablanc C, Martin O. Automatic control during hand reaching at undetected two-dimensional target displacements. J Neurophysiol 1992;67:455-69.

Prado J, Clavagnier S, Otzenberger H, Scheiber C, Kennedy H, Perenin MT. Two cortical systems for reaching in central and peripheral vision. Neuron 2005;48:849-58. 
Scherberger H, Andersen RA. Target selection signals for arm reaching in the posterior parietal cortex. J Neurosci 2007;27:2001-12.

Scherberger H, Jarvis MR, Andersen RA. Cortical local field potential encodes movement intentions in the posterior parietal cortex. Neuron 2005;46:347-54.

Smid HG, Mulder G, Mulder LJ. The continuous flow model revisited: perceptual and central motor aspects. Electroencephalogr Clin Neurophysiol Suppl 1987;40:270-8.

Snyder LH, Batista AP, Andersen RA. Coding of intention in the posterior parietal cortex. Nature 1997;386:167-70.

Tombini M, Zappasodi F, Zollo L, Pellegrino G, Cavallo G, Tecchio F, et al. Brain activity preceding a 2D manual catching task. Neuroimage 2009;47:1735-46.

Toro C, Deuschl G, Thatcher R, Sato S, Kufta C, Hallett M. Event-related desynchronization and movement-related cortical potentials on the ECoG and EEG. Electroencephalogr Clin Neurophysiol 1994;93:380-9.

Van Der Werf J, Jensen O, Fries P, Medendorp WP. Neuronal synchronization in human posterior parietal cortex during reach planning. J Neurosci 2010;30:1402-12.

Van Winsum W, Sergeant J, Geuze R. The functional significance of event-related desynchronization of alpha rhythm in attentional and activating tasks. Electroencephalogr Clin Neurophysiol 1984;58:519-24.

Virji-Babul N, Moiseev A, Cheung T, Weeks D, Cheyne D, Ribary U. Spatial-temporal dynamics of cortical activity underlying reaching and grasping. Hum Brain Mapp 2010;31:160-71. 


\section{Figure Legends}

\section{Figure 1}

Electroencephalography (EEG) electrode placement. Localization of the 5 areas of interest: left fronto-central (F3, FC1, FC3, C1, C3), right fronto-central (F4, FC2, FC4, $\mathrm{C} 2, \mathrm{C} 4)$, midline (Fz, FCz, Cz), left parietal area (CP3, P3, P7, PO3, PO7), right parietal area $(\mathrm{CP} 4, \mathrm{P} 4, \mathrm{P} 8, \mathrm{PO} 4, \mathrm{PO} 8)$.

\section{Figure 2}

Mean event-related desynchronization (ERD) values across all subjects. $\boldsymbol{A}$ : ERD value at $\mathrm{DC}-30 \mathrm{~Hz}$ for $3000 \mathrm{~ms}$ before the movement onset, a clear alpha-band ERD is observed before the reaching movement. Upper row: reaching, lower row: wrist extension, left column: left hand movement, right column: right hand movement. The color format is as follows: warmer (red) colors, power increase relative to baseline; cooler (blue) colors, power decrease. B: ERD laterality for left hand movement (left column) and right hand movement (right column) across all subjects. A significant ERD lateralization toward the contralateral hemisphere to the moving hand was present before the right hand movement as compared with the left hand movement (Significant interactions: Hand $\times$ Region $(\mathrm{F}(4,52)=5.210, \mathrm{P}=0.001))$. $\boldsymbol{C}$ : Mean lateralized ERD index (LERDI) for reaching (a white circle) and wrist extension (a gray circle) across all subjects. Error bars represent \pm 1 standard deviation of the mean. Negative values indicate the ERD preference for the contralateral hand; positive values indicate the ipsilateral hand; $* \mathrm{P}<0.05, * * \mathrm{P}<0.001$. D: Mean onset of the $12-\mathrm{Hz}$ ERD in the contralateral parietal area before the movement onset (a white bar: reaching, a gray bar: wrist extension).

\section{Figure 3}

Mean topographic representations (4 maps on the left) of the 12-Hz ERD from 1050 to $550 \mathrm{~ms}$ before the movement onset and of the posterior dominant rhythm (PDR) power at $10 \mathrm{~Hz}$ (1 map on the right) across all subjects. The black dots indicate the EEG electrodes. The color indicates power differences from the baseline in log scale. 
Figure 1

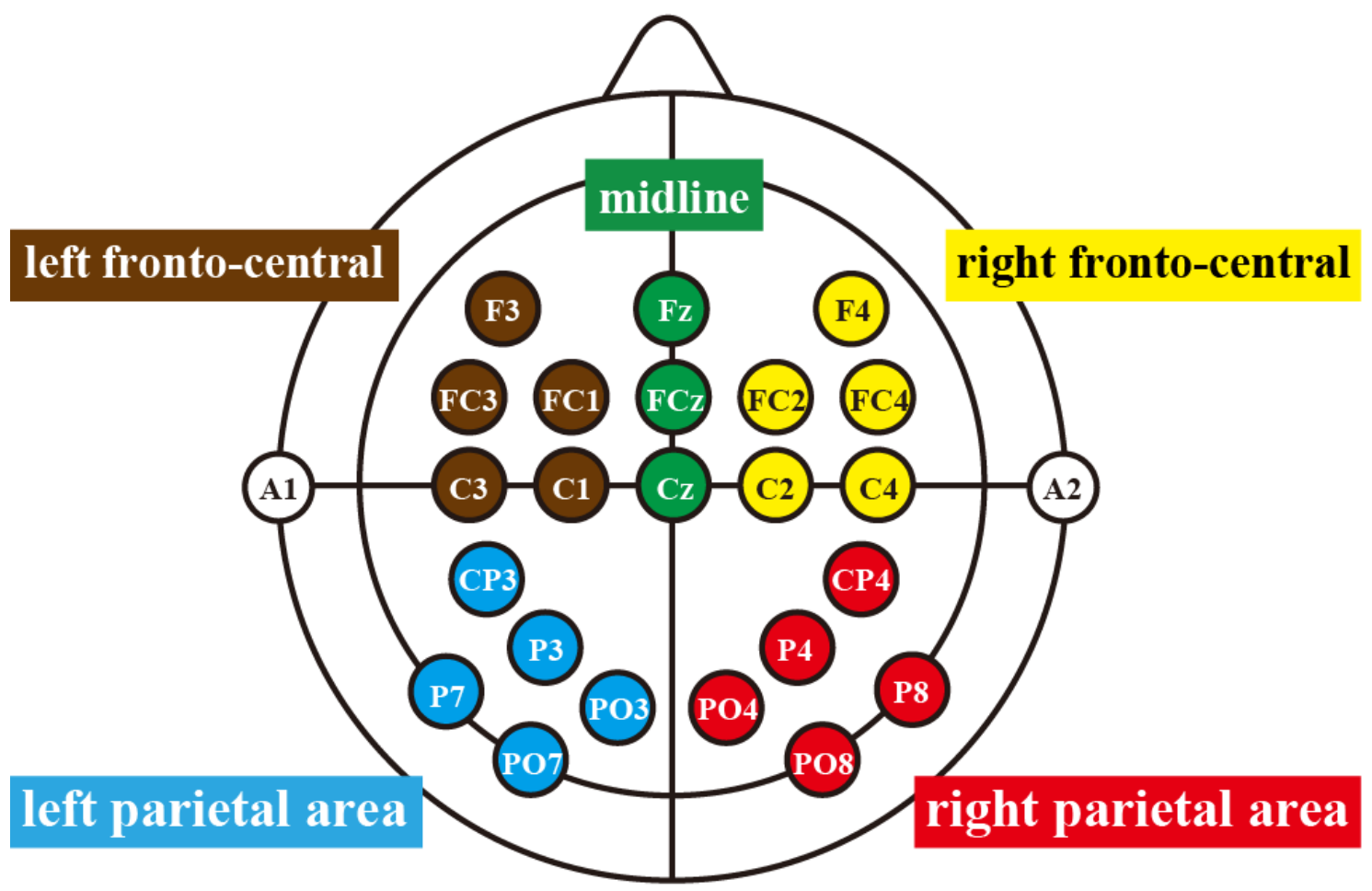


Figure 2

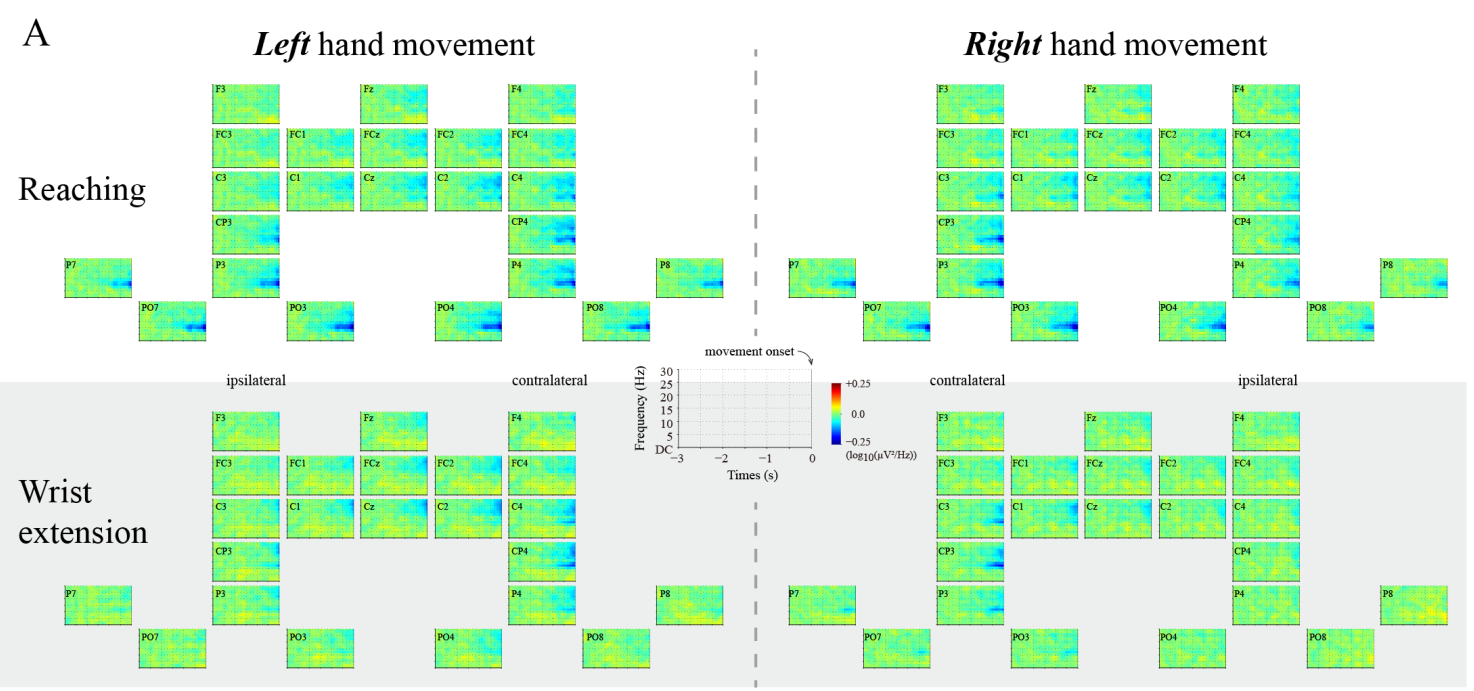

B

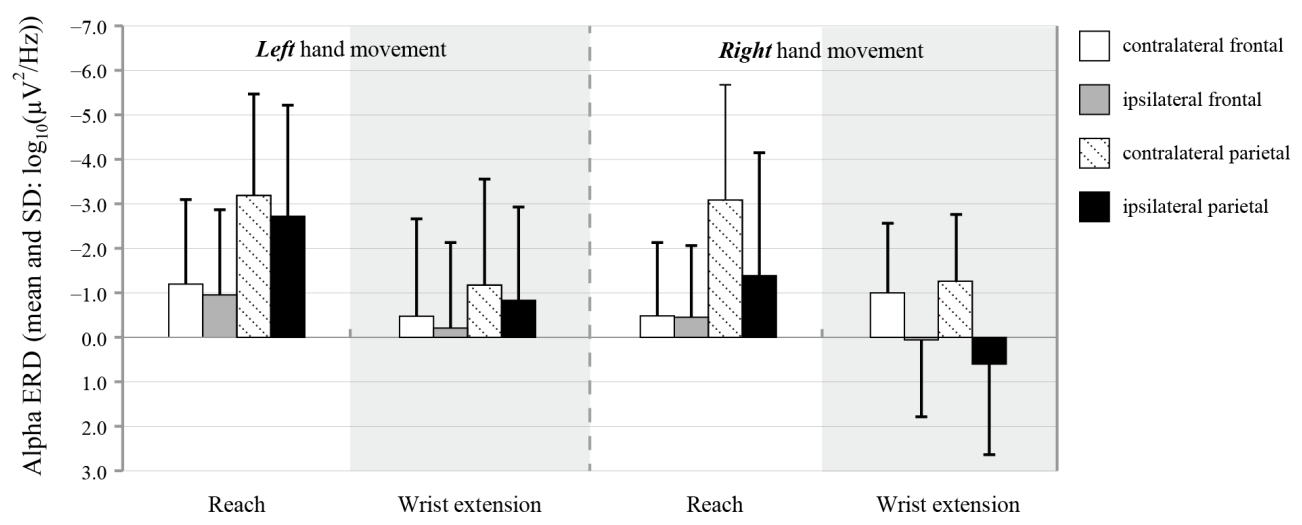

C

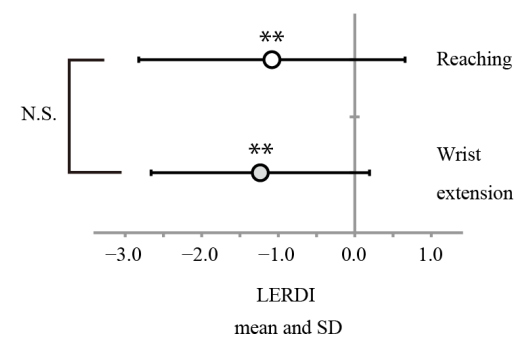

D

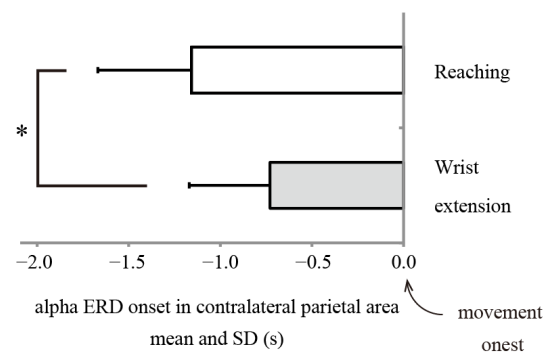


Figure 3

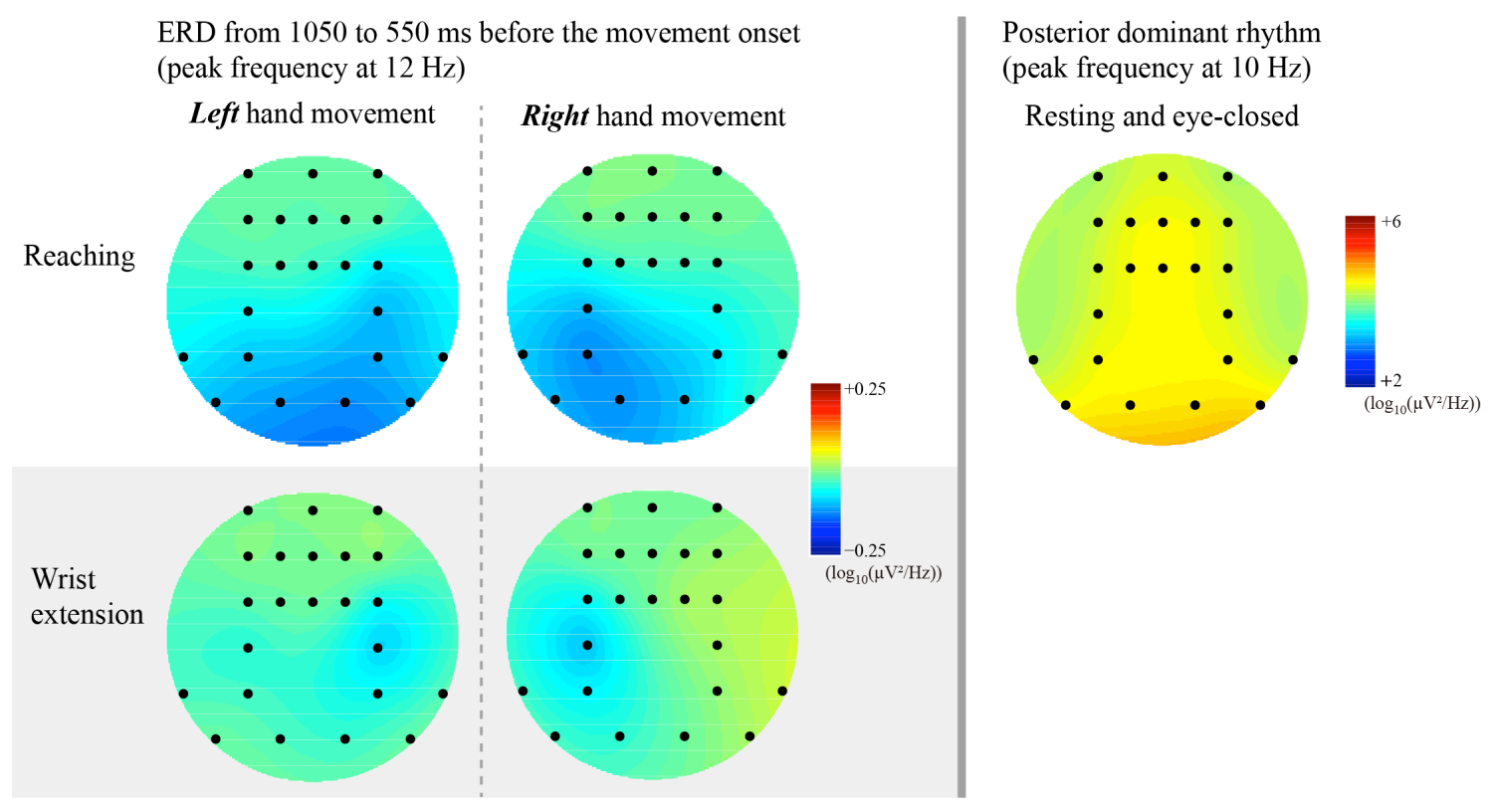

